\title{
Interactions between Host Immunity and Skin-Colonizing Staphylococci: No Two Siblings Are Alike
}

\author{
Young Joon Park ${ }^{1}$, Chae Won Kim ${ }^{2}$ and Heung Kyu Lee 1,2,3,*(D) \\ 1 Graduate School of Medical Science and Engineering, Korea Advanced Institute of Science and \\ Technology (KAIST), Daejeon 34141, Korea; youngjoon.park@kaist.ac.kr \\ 2 Biomedical Science and Engineering Interdisciplinary Program, KAIST, Daejeon 34141, Korea; \\ chaewon.kim@kaist.ac.kr \\ 3 KAIST Institute for Health Science and Technology, KAIST, Daejeon 34141, Korea \\ * Correspondence: heungkyu.lee@kaist.ac.kr; Tel.: +82-42-350-4241; Fax: +82-42-350-4240
}

Received: 29 January 2019; Accepted: 4 February 2019; Published: 7 February 2019

\begin{abstract}
As the outermost layer of the body, the skin harbors innumerable and varied microorganisms. These microorganisms interact with the host, and these interactions contribute to host immunity. One of the most abundant genera of skin commensals is Staphylococcus. Bacteria belonging to this genus are some of the most influential commensals that reside on the skin. For example, colonization by Staphylococcus aureus, a well-known pathogen, increases inflammatory responses within the skin. Conversely, colonization by Staphylococcus epidermis, a coagulase-negative staphylococcal species that are prevalent throughout the skin, can be innocuous or beneficial. Thus, manipulating the abundance of these two bacterial species likely alters the skin microbiome and modulates the cutaneous immune response, with potential implications for various inflammation-associated skin diseases. Importantly, before researchers can begin manipulating the skin microbiome to prevent and treat disease, they must first fully understand how these two species can modulate the cutaneous immune response. In this review, we discuss the nature of the interactions between these two bacterial species and immune cells within the skin, discussing their immunogenicity within the context of skin disorders.
\end{abstract}

Keywords: cutaneous immunity; microbiome; Staphylococcus spp., T cells; Staphylococcus aureus; Staphylococcus epidermis; commensals; atopic dermatitis

\section{Introduction}

The skin is the largest organ of the body and is inhabited by millions of commensals, including bacteria, fungi, and viruses. Despite their abundance, whether these microorganisms benefit the host is unclear. Recent technological advances such as genome sequencing have expanded our understanding of the composition and function of the skin microbiome. We now know that the microorganisms which reside in our body fall on a spectrum from 'good' to 'troublesome' residents. Specifically, beneficial microflora (the 'good' residents) usually cause no secondary infections, but cannot be eliminated as causes of illness [1]. Alternatively, troublesome residents are generally considered pathogenic but can sometimes aid the host by preventing colonization by other microorganisms.

In the skin, one of the most studied troublesome residents is Staphylococcus aureus. These bacteria have long been notorious pathogens. S. aureus belong to the genus Staphylococcus, which is comprised of fifteen unique species that are differentiated based on molecular data [2]. Whether S. aureus should be considered a commensal is controversial; however, approximately $30 \%$ of the human population carries asymptomatic S. aureus in their nose [3]. Healthy control skin also carries 
S. aureus, indicating that the presence of S. aureus is not always directly associated with disease [4]. Comprehensive research evaluating different species of Staphylococcus has shown that species other than S. aureus can also interact with hosts. One such species that has been the subject of recent research is Staphylococcus epidermidis. As members of the same genus, S. aureus and S. epidermidis share key phenotypic properties [2]. However, S. epidermidis belongs to a group known as coagulase-negative staphylococci (CoNS). As the name suggests, S. epidermidis do not possess coagulase, a blood-clotting enzyme, which distinguishes these bacteria from S. aureus. In addition, unlike S. aureus, S. epidermidis are generally good residents. They can also interact with the environment, and these interactions often benefit host immunity. It is interesting that although these two bacteria belong to the same genus, their effects are antagonistic. Several proteins expressed by these microorganisms, and their immunogenicity, have been uncovered. However, more research is needed regarding the biochemical characteristics and functions of these microorganisms. In this review, we provide an overview of what is currently known regarding the effects of skin colonization by S. epidermidis and S. aureus on protein production and host cutaneous immunity.

\section{S. aureus}

Before addressing the interactions between S. aureus and local immunity, we must first note that the interactions as a member of the skin microbiome differ from deep or systemic staphylococcal infections. Indeed, interactions as a resident microorganism are confined to only a minor breach of the cutaneous barrier. Soft tissue staphylococcal infections and biofilm infections on implanted medical devices are beyond the scope of this review.

As a resident microorganism, $S$. aureus colonization is associated with various skin diseases, including atopic dermatitis (AD). Even before DNA sequencing technology emerged, S. aureus was known to be more frequent in AD patients [5]. Real-Time PCR (RT-PCR) studies confirmed the increased prevalence of $S$. aureus in the skin lesions of $\mathrm{AD}$ patients [6]. AD is generally considered an inflammatory skin disorder with Th2 skewing. Thus, the relationship between S. aureus, inflammation, and Th2 responses has been brought to the attention of those who study S. aureus and AD (Figure 1).

Importantly, S. aureus colonization noticeably affects cutaneous immunity. In barrier-disrupted murine skin, S. aureus colonization increases the expression of the pro-inflammatory cytokines IL- $1 \beta$, IL-6, and TNF- $\alpha$ [7]. Upon entering the dermis, S. aureus increases mRNA expression of IL-4, IL-13, CXCL2, TSLP, IL-17, and IL-22, but decreases expression of cathelicidin, an antimicrobial peptide [8]. This penetration is enhanced by protease-induced S. aureus. S. aureus can also modulate cutaneous immunity through production of staphylococcal serine protease-like proteins (Spls). Spls are a group of secreted bacterial proteases that are currently known for their allergenic properties in mice and humans. When stimulated with Spls, human peripheral blood T cells produce significant amounts of Th2, but not Th1/Th17, cytokines [9]. One such Spl, SPID, induces type 2 airway responses in an IL-33-dependent manner [10]. Thus, Spls may underlie the association between S. aureus colonization and Th2 responses in AD.

Unlike S. epidermidis, S. aureus secrete toxins. One such toxin, $\delta$-toxin, is known to promote Th2 responses [11]. Skin colonization with $S$. aureus, but not with a mutant deficient in $\delta$-toxin, promoted immunoglobulin-E (IgE) and interleukin-4 production, as well as inflammatory skin disease. $\delta$-toxin induces degranulation of dermal mast cells and increases IgE, which further enhances mast cell degranulation. This cycle may also contribute to Th2 skewing.

Furthermore, S. aureus membrane components and diacylated lipopeptide induce keratinocyte production of thymic stromal lymphopoietin (TSLP), which is an important initiator of AD [12]. S. aureus cell wall components also downregulate IP-10, trigger activation of MAPK, p38, and ERK, and inhibit STAT1 signaling in monocytes, all of which may contribute to the abrogation of Th1 cell-recruiting chemokines [3]. 


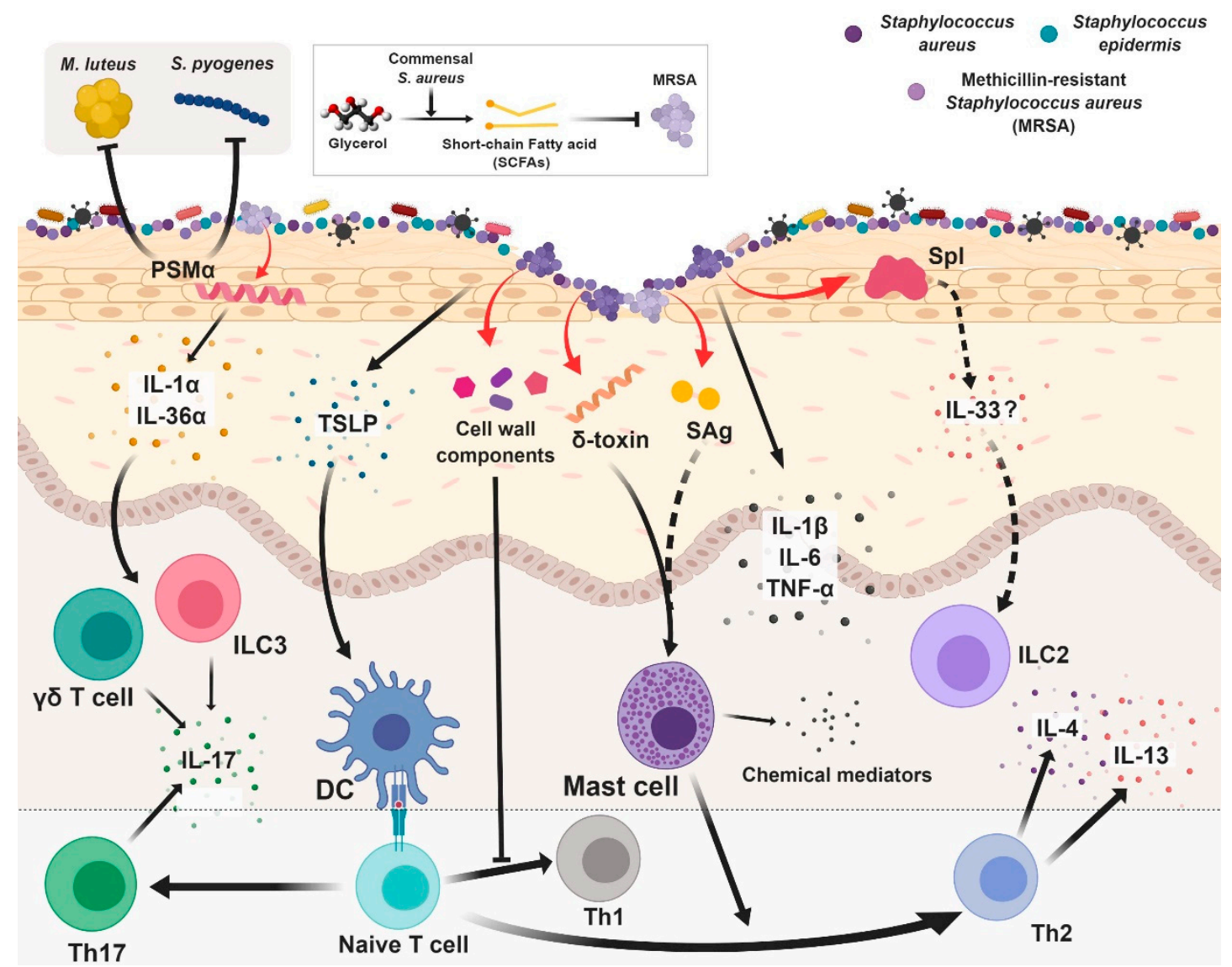

Figure 1. Interaction between Staphylococcus aureus and skin immune system. The skin immune system comprises a complex network of cells. Because keratinocytes serve as sensors, phenol-soluble modulin $\alpha(\operatorname{PSM} \alpha)$ are capable of inducing epidermal keratinocytes to produce IL-1 and IL-36, even without epidermal disruption. T cells and innate lymphoid cells (ILC3) containing the IL-36 receptor recognize IL-13 and secrete IL-17, leading to inflammation. Another peptide belonging to the PSM family, $\delta$-toxin, promotes Th2 responses by activating mast cells. It is likely that staphylococcal superantigens (SAgs) are also capable of mast cell activation. Thymic stromal lymphopoietin (TSLP) can drive Th2 skewing via dendritic cells (DCs). Cell wall components from S. aureus inhibit Th1 responses. S. aureus also possesses probiotic properties. S. aureus shows antimicrobial activity against other bacteria, such as M. luteus and S. pyogenes. Commensal S. aureus inhibits methicillin-resistant S. aureus (MRSA) using short-chain fatty acids (SCFAs) fermented from glycerol. Red arrows indicate bacteria-originated materials. T-bar indicates inhibitory activity.

S. aureus also express superantigens (SAgs), including toxic shock syndrome toxin-1 and staphylococcal enterotoxin, that cross-bridge major histocompatibility class II molecules on antigen presenting cells and the $\mathrm{T}$ cell receptor on $\mathrm{T}$ cells. This binding strongly promotes $\mathrm{T}$ cell proliferation the release of Th1 cytokines. When applied to human skin, staphylococcal enterotoxin B (SEB) promotes cutaneous $\mathrm{T}$ cell accumulation and an AD-like response [13]. Whether this is a Th1 or Th2 response is unclear, but SAgs can interact directly with mast cells and induce T cells to secrete IL-31 [14]. IL-31 inhibits keratinocyte differentiation, downregulates filaggrin expression, and causes an itching sensation, all of which are implicated in AD development [14,15].

Finally, recent reports indicate that $S$. aureus colonization may also contribute to psoriasis and new-onset pediatric AD, both of which are associated with a Th17 rather than a Th2 response [16]. In these studies, epicutaneous $S$. aureus exposure for one week induces skin inflammation. S. aureus produces phenol-soluble modulin $\alpha$ (PSM $\alpha$ ), which triggers IL-36R/MyD88 signaling. Consequently, IL-36 from epidermal keratinocytes stimulates T cells, which generate IL-17 $[17,18]$. As human AD 
skin has increased IL-36 $\alpha / \gamma$ transcripts and an increased number of Th17 cells [19,20], excess PSMs from S. aureus may also be responsible for Th17-polarized skin inflammation.

S. aureus PSMs, nonetheless, may be of advantage to the host. PSM derivatives from PSM $\alpha 1$ and $\alpha 2$ display antimicrobial activity against $M$. luteus and S. pyogenes, a component of the normal flora and a highly pathogenic bacterium, respectively. Contrary to expected results, lantibiotics (lanthionine-containing peptide antibiotics produced by bacteria) did not show antimicrobial activity in the study [21].

Recently, a commensal S. aureus strain isolated from human perinasal skin demonstrated the ability of fermenting glycerol to release short-chain fatty acids (SCFAs), which are known for their bactericidal activity. USA300, a representative methicillin-resistant strain of $S$. aureus, does not possess such ability. When commensal S. aureus and methicillin-resistant S. aureus (MRSA) with/without 2\% glycerol were applied onto the skin of wounded mice, the number of MRSA was significantly less in wounds inoculated with bacteria and glycerol than in those inoculated without glycerol. Furthermore, immunization of mice with recombinant $\alpha$-hemolysin from commensal $S$. aureus yielded high antibody titers $(>1: 512,000)$, which provided protection against MRSA skin infection. These data suggest that commensal S. aureus bacteria may provide benefits to the host by maintaining host probiotic activity, whereas this characteristic has not been evolutionarily conserved in MRSA [22].

\section{S. epidermidis}

Compared to S. aureus, S. epidermidis is less pathogenic, and colonization by S. epidermidis may even benefit the host local immune system (Summarized in Figure 2). S. epidermidis produces small molecules that activate Toll-like receptor (TLR) 2. Exposure of cultured human keratinocytes to a sterile nontoxic small molecule $(<10 \mathrm{kDa})$ isolated from S. epidermidis-conditioned culture medium (SECM) increases mRNA expression of antimicrobial peptides (i.e., human beta-defensin 2 [hBD2] and hBD3). It also enhances the capacity of cell lysates to inhibit group A Streptococcus (GAS) and promotes S. aureus growth [23]. In mice, SECM administration decreases the susceptibility to GAS infection, confirming that this effect also occurs in vivo. Importantly, this process is TLR2-dependent, as treatment with a TLR2-neutralizing antibody blocks hBD induction, and TLR2-deficient mice do not show induction of mBD4 (mouse ortholog of hBD2). Lipoteichoic acid (LTA), produced by S. epidermidis and recognized by TLR2, has proven to be effective in suppressing inflammation. Following skin injury, LTA-induced TLR2 signaling inhibits TLR3-mediated skin inflammation. Specifically, TLR3 signaling is suppressed by induction of TNF receptor-associated factor-1 (TRAF1), a negative regulatory factor that suppresses keratinocyte cytokines (e.g., TNF- $\alpha$ ) released via the TLR3 signaling cascade [24]. Lipopeptide 78 (LP78) is another molecule from SECM that has been shown to suppress skin inflammation. LP78 activates TLR2-SRC signaling in normal human epidermal keratinocytes (NHEKs) to induce $\beta$-catenin phosphorylation. Phospho- $\beta$-catenin translocates into the nucleus to bind to peroxisomal proliferator-activated receptor gamma (PPAR $\gamma$ ), disrupting the interaction between p65 and PPAR $\gamma$. Dissociation of p65 from PPAR $\gamma$ reduces the expression of TLR3-induced inflammatory cytokines in skin wounds of normal and diabetic mice, which correlates with accelerated wound healing [25].

S. epidermidis also produces PSMs (PSM $\gamma$ and PSM $\delta$ ), which directly induce lipid vesicle leakage and antimicrobial action against skin pathogens such as S. aureus [26]. PSMs reduce GAS selectively without affecting S. epidermidis growth. Finally, synthetic PSM $\delta$ not only binds to and increases the killing capacity of neutrophil extracellular traps, it also binds to host-derived AMPS (i.e., LL-37, CRAMP, hBD2 and hBD3) and enhances their endogenous antimicrobial activity [27]. 


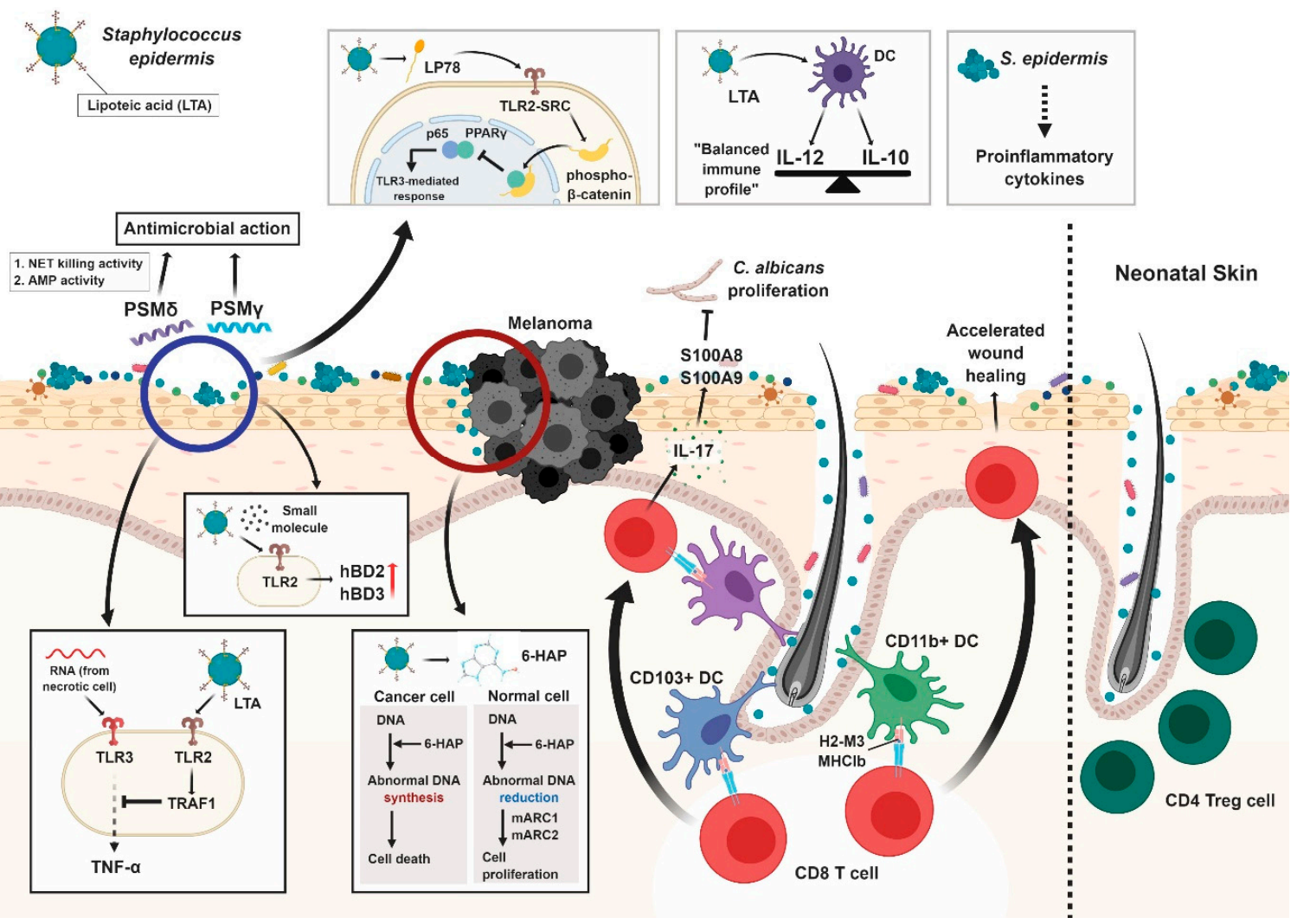

Figure 2. Interaction between Staphylococcus epidermis and skin immune system. When skin is injured, lipoteichoic acid (LTA) from the skin commensal S. epidermidis inhibits inflammatory cytokine production via inhibition of TLR3 signaling by TRAF1 activated through TLR2. S. epidermidis also produces small molecules that increase anti-microbacterial peptides through TLR2, which in turn inhibits pathogen growth. Lipopeptide 78 activates TLR2-SRC signaling in keratinocytes to induce $\beta$-catenin phosphorylation. Dissociation between $\mathrm{p} 65$ and PPAR $\gamma$ due to phospho- $\beta$-catenin binding reduces TLR3-mediated inflammation. Phenol-soluble modulin $\gamma(\operatorname{PSM} \gamma)$ and PSM $\delta$ from S. epidermidis also have anti-microbacterial activity. 6-HAP, a nucleobase analogue capable of selective tumor growth suppression was recently found to be produced by $S$. epidermidis. Cancer cells did not have sufficient mARC1 and mARC2 to reduce 6-HAP, leading to cell death. CD103+ dendritic cells (DCs) and CD11b+ DCs cooperate in tuning $S$. epidermidis-specific CD8+ T cells to protect from pathogen infection via upregulation of alarmins (S100A8 and S100A9). The commensal-specific CD8+ T cell response is induced by non-classical MHC class I molecules ( $\mathrm{H} 2-\mathrm{M} 3 \mathrm{MHCIb})$, and is immunoregulatory and tissue-repairing in nature. Dendritic cells (DCs) exposed to LTA preparations from S. epidermidis (epi-LTA) show an IL-10-balanced immune profile, with the ratio of IL-12p70 to IL-10 being close to 1; likewise, naïve CD4+ T cells primed by epi-LTA produce lower amounts of IFN- $\gamma$ and IL-17. In neonatal skin, S. epidermidis is critical for the accumulation of CD4+ regulatory T cells, establishing tolerance to skin commensals. Even in low amounts, the inflammatory cytokines induced by S. epidermidis can lead to various consequences, such as sepsis, bronchopulmonary dysplasia, white matter injury, necrotizing enterocolitis, and retinopathy of prematurity.

The Gallo group recently discovered that some strains of $S$. epidermidis also produce 6-N-hydroxyaminopurine (6-HAP), a molecule that inhibits DNA polymerase activity. In the context of cancer, 6-HAP inhibits tumor cell proliferation in vitro and B16F10 melanoma growth in vivo [28]. This anti-proliferative activity of 6-HAP is mediated by mitochondrial amidoxime reducing components (mARC1 and mARC2). Importantly, silencing $m A R C$ with small interfering RNA (siRNA) partially inhibits sensitivity to 6-HAP in non-transformed human keratinocytes. Alternatively, colonization with a 6-HAP-producing S. epidermidis strain reduces the incidence of ultraviolet-induced tumors in mice. Thus, further study is needed to examine whether the loss of specific S. epidermidis strains increases 
skin cancer risk in humans, or if increasing S. epidermidis skin colonization prevents cancer. Ongoing research is exploring how dysbiosis affects cancer. It has been demonstrated that inflammation induced by dysbiosis promotes carcinogenesis $[29,30]$. Other observations from the intestinal microbiome suggest that microbes may suppress tumor growth by producing short-chain free fatty acids $[29,31]$. This study clearly shows that a skin bacterium can protect against neoplasia. S. epidermidis or other skin commensals may interact with the tumor environment.

S. epidermidis colonization also affects adaptive cutaneous immunity (Figure 2). Indeed, S. epidermidis induces IL-17A+/CD8 $+\mathrm{T}$ cell homing to the epidermis and enhances barrier immunity [32]. Naik et al. demonstrated that the commensal-driven CD8+ T cell response is antigen specific, and is mediated by a particular subset of dendritic cells (CD103+ dendritic cells). In turn, these IL17A+ CD8+ cells promote skin innate immune responses that protect from cutaneous Candida albicans infections. Whether S. aureus growth was also suppressed in prior association with S. epidermidis was not discussed in the study.

A recent study by the Belkaid group also showed that a specific clade (A20) of S. epidermidis induces CD8+ T cell responses. This clade is highly prevalent on human skin, and topical application of the NIHLM087 (from A20 clade) to the skin of non-human primates increases the absolute number of Tc17 cells, but not CD4+ T cells. Hence, the ability of S. epidermidis to promote skin CD8+ T cell responses is conserved in non-human primates and likely exists in humans. Furthermore, the researchers specified the characteristics of these CD8+ T cells. Using MHC class I K $\mathrm{K}^{\mathrm{b}} \mathrm{b}^{\mathrm{-}-}$ - dendritic cells from $\mathrm{H} 2-\mathrm{M}^{-/}{ }^{-}$mice that lack MHC class Ia alleles, they demonstrated that $S$. epidermidis-specific CD8+ T cells are confined to MHC class $\mathrm{Ib}$, particularly H2-M3 MHC class Ib. H2-M3-resticted CD8+ $\mathrm{T}$ cells are specific to $\mathrm{S}$. epidermidis-derived fMet peptide ligands. RNA sequencing also revealed the immunoregulatory and tissue-repair signatures of these CD8+ T cells. In a skin wounding model, S. epidermidis-induced CD8+ T cells promoted more rapid epidermal keratinocyte progression, leading to accelerated wound healing.

S. epidermidis is also involved in immune tolerance. Tolerance to commensals is crucial for maintaining immune homeostasis at barrier sites. Scharschmidt et al. has created an S. epidermidis strain that expresses a model $\mathrm{T}$ cell antigen [33]. Using this strain, they discovered that commensal antigens are recognized both locally and systemically across an intact skin barrier. Tolerance to skin commensal bacteria is established in response to neonatal, but not adult, colonization. Tolerance results due to the abrupt accumulation of CD4+ Treg cells between postnatal days 6 and 13. The accumulation of Treg cells is tissue (skin)-specific.

When murine dendritic cells (DCs) were exposed to LTA preparations from S. epidermidis (epi-LTA), the ratio of IL-12p70 to IL-10 was close to 1 (IL-10-balanced immune profile), whereas LTA preparations from S. aureus (aureus-LTA) induced DCs to produce much higher levels of IL12-p70 (ratio >3). Naïve CD4+ T cells primed by epi-LTA produced significantly lower amounts of IFN- $\gamma$ and IL-17 compared with those primed by aureus-LTA. The IL-10-balanced immune profile of DCs exposed to epi-LTA was preserved in the presence of the Th2 hallmark cytokine IL-4. Although this should be tested in vivo, we can assume that $S$. epidermidis LTA does not contribute to enhanced cutaneous inflammation in an IL-4-rich environment, but rather regulates the inflammatory response [34]. Taken together, these studies suggest that modulation of S. epidermidis can be used to prevent skin diseases.

Despite the favorable results that have been reported for S. epidermidis, it remains a major nosocomial pathogen [35]. Risk factors for infection include the presence of indwelling implants, immunosuppression, and increasing antibiotic resistance [36,37]. S. epidermidis is also a predominant pathogen of sepsis in preterm infants. Setting aside nosocomial environmental factors, S. epidermidis alone is capable of inducing pro-inflammatory cytokine production (e.g., TNF- $\alpha$, IL-6, and IL-8). Data concerning S. epidermidis-induced inflammatory mediators are predominantly based on in vitro experiments and should be studied further in vivo [38].

Multiple studies have reported that the S. epidermidis isolated from infections belong to a subset of those found on the skin surface [39]. Using a genome-wide association study (GWAS) approach, 
61 genes containing infection-associated genetic elements ( $k$-mers) that correlate with in vitro variations in known pathogenicity traits (i.e., biofilm formation, cell toxicity, interleukin-8 production, and methicillin resistance) were identified. The authors concluded from the data analysis that the disease-causing S. epidermidis appears to represent a pathogenic sub-population. These organisms are likely to have acquired genetic elements and related phenotypes that promote infection [40].

Unfortunately, identifying the specific factor or event that drives the transition from harmless commensal to pathogenic factor in skin disease, especially those other than infection, is difficult. Hon et al. examined 100 adolescents with $\mathrm{AD}$ and found that S. epidermidis was present in severely AD-affected skin lesions, with a positive association to SCORing Atopic Dermatitis (SCORAD) [41]. Alternatively, another recent study by Byrd et al. showed that patients with more mild disease had more S. epidermidis detected in flares, and that those with severe disease were predominantly colonized by clonal S. aureus strains [42]. Thus, further detailed studies investigating the pro-inflammatory capacity of S. epidermidis and its effects on local inflammation and immune priming are needed to determine the role of $S$. epidermidis in inflammatory skin diseases.

\section{Concluding Remarks}

Although S. aureus and S. epidermidis are not the only Staphylococcal species present in the skin, these bacteria are particularly important because they are the most recognized in pathogenic and healthy skin, respectively. Manipulation of these bacteria is likely to significantly affect skin microbiota-host interactions. One way of manipulating the skin microbiota is to introduce specific strains to subjects by topical application. A study has shown that such introduction successfully decreased colonization by $S$. aureus. The result was due to the antimicrobial peptides produced from CoNS [43].

Although these two bacteria share substantial phenotypic properties, their effects on the skin microbiota are contradictory. These differential effects are likely related to different structural adaptations, but those are currently unknown. Additionally unknown are the detailed mechanisms underlying how a specific subset of antigen presenting cells and their epitopes stimulate conventional $\mathrm{T}$ cells. Importantly, the skin is a reservoir for tissue-resident memory $\mathrm{T}$ cells, which are distinct from recirculating central and effector memory $\mathrm{T}$ cells and mucosal-associated invariant $\mathrm{T}$ cells. Thus, it is plausible that $S$. aureus and/or S. epidermidis affect cutaneous immunity and the homeostatic response through these T cells. Studies evaluating how manipulating these microbes affects various subsets of $\mathrm{T}$ cells and long-term local immunity should be performed to discover novel therapeutic strategies. As such, numerous studies are required to understand the complex dialogue between cutaneous microorganisms and host immunity. For now, detailed characterization of the immunogenicity of S. aureus and S. epidermidis is essential.

Author Contributions: Y.J.P., C.W.K. and H.K.L. wrote the manuscript.

Funding: This study was supported by the National Research Foundation of Korea (NRF-2018M3A9H3024611) funded by the Ministry of Science and ICT of Korea. Young Joon Park is a recipient of a Global Ph.D. fellowship supported by the National Research Foundation of Korea (NRF-2017H1A2A1045742).

Acknowledgments: The authors would like to thank the members of the Laboratory of Host Defenses for their helpful advice and discussions.

Conflicts of Interest: The authors declare no conflict of interest.

\section{References}

1. Hand, T.W. The Role of the Microbiota in Shaping Infectious Immunity. Trends Immunol. 2016, 37, $647-658$. [CrossRef] [PubMed]

2. Lamers, R.P.; Muthukrishnan, G.; Castoe, T.A.; Tafur, S.; Cole, A.M.; Parkinson, C.L. Phylogenetic relationships among Staphylococcus species and refinement of cluster groups based on multilocus data. BMC Evol. Biol. 2012, 12, 171. [CrossRef] [PubMed] 
3. Krismer, B.; Weidenmaier, C.; Zipperer, A.; Peschel, A. The commensal lifestyle of Staphylococcus aureus and its interactions with the nasal microbiota. Nat. Rev. Microbiol. 2017, 15, 675-687. [CrossRef] [PubMed]

4. Totte, J.E.; van der Feltz, W.T.; Hennekam, M.; van Belkum, A.; van Zuuren, E.J.; Pasmans, S.G. Prevalence and odds of Staphylococcus aureus carriage in atopic dermatitis: A systematic review and meta-analysis. Br. J. Dermatol. 2016, 175, 687-695. [CrossRef]

5. Higaki, S.; Morohashi, M.; Yamagishi, T.; Hasegawa, Y. Comparative study of staphylococci from the skin of atopic dermatitis patients and from healthy subjects. Int. J. Dermatol. 1999, 38, 265-269. [CrossRef] [PubMed]

6. Tauber, M.; Balica, S.; Hsu, C.Y.; Jean-Decoster, C.; Lauze, C.; Redoules, D.; Viode, C.; Schmitt, A.M.; Serre, G.; Simon, M.; et al. Staphylococcus aureus density on lesional and nonlesional skin is strongly associated with disease severity in atopic dermatitis. J. Allergy Clin. Immunol. 2016, 137, 1272-1274.e3. [CrossRef] [PubMed]

7. Wanke, I.; Skabytska, Y.; Kraft, B.; Peschel, A.; Biedermann, T.; Schittek, B. Staphylococcus aureus skin colonization is promoted by barrier disruption and leads to local inflammation. Exp. Dermatol. 2013, 22, 153-155. [CrossRef]

8. Nakatsuji, T.; Chen, T.H.; Two, A.M.; Chun, K.A.; Narala, S.; Geha, R.S.; Hata, T.R.; Gallo, R.L. Staphylococcus aureus Exploits Epidermal Barrier Defects in Atopic Dermatitis to Trigger Cytokine Expression. J. Investig. Dermatol. 2016, 136, 2192-2200. [CrossRef]

9. Stentzel, S.; Teufelberger, A.; Nordengrun, M.; Kolata, J.; Schmidt, F.; van Crombruggen, K.; Michalik, S.; Kumpfmuller, J.; Tischer, S.; Schweder, T.; et al. Staphylococcal serine protease-like proteins are pacemakers of allergic airway reactions to Staphylococcus aureus. J. Allergy Clin. Immunol. 2017, 139, 492-500.e8. [CrossRef]

10. Teufelberger, A.R.; Nordengrun, M.; Braun, H.; Maes, T.; De Grove, K.; Holtappels, G.; O’Brien, C.; Provoost, S.; Hammad, H.; Goncalves, A.; et al. The IL-33/ST2 axis is crucial in type 2 airway responses induced by Staphylococcus aureus-derived serine protease-like protein D. J. Allergy Clin. Immunol. 2018, 141, 549-559.e7. [CrossRef]

11. Nakamura, Y.; Oscherwitz, J.; Cease, K.B.; Chan, S.M.; Munoz-Planillo, R.; Hasegawa, M.; Villaruz, A.E.; Cheung, G.Y.; McGavin, M.J.; Travers, J.B.; et al. Staphylococcus delta-toxin induces allergic skin disease by activating mast cells. Nature 2013, 503, 397-401. [CrossRef]

12. Vu, A.T.; Baba, T.; Chen, X.; Le, T.A.; Kinoshita, H.; Xie, Y.; Kamijo, S.; Hiramatsu, K.; Ikeda, S.; Ogawa, H.; et al. Staphylococcus aureus membrane and diacylated lipopeptide induce thymic stromal lymphopoietin in keratinocytes through the Toll-like receptor 2-Toll-like receptor 6 pathway. J. Allergy Clin. Immunol. 2010, 126, 985-993.e3. [CrossRef] [PubMed]

13. Skov, L.; Olsen, J.V.; Giorno, R.; Schlievert, P.M.; Baadsgaard, O.; Leung, D.Y. Application of Staphylococcal enterotoxin $\mathrm{B}$ on normal and atopic skin induces up-regulation of $\mathrm{T}$ cells by a superantigen-mediated mechanism. J. Allergy Clin. Immunol. 2000, 105, 820-826. [CrossRef] [PubMed]

14. Cornelissen, C.; Marquardt, Y.; Czaja, K.; Wenzel, J.; Frank, J.; Luscher-Firzlaff, J.; Luscher, B.; Baron, J.M. IL-31 regulates differentiation and filaggrin expression in human organotypic skin models. J. Allergy Clin. Immunol. 2012, 129, 426-433.e8. [CrossRef] [PubMed]

15. Geoghegan, J.A.; Irvine, A.D.; Foster, T.J. Staphylococcus aureus and Atopic Dermatitis: A Complex and Evolving Relationship. Trends Microbiol. 2018, 26, 484-497. [CrossRef] [PubMed]

16. Chang, H.W.; Yan, D.; Singh, R.; Liu, J.; Lu, X.; Ucmak, D.; Lee, K.; Afifi, L.; Fadrosh, D.; Leech, J.; et al. Alteration of the cutaneous microbiome in psoriasis and potential role in Th17 polarization. Microbiome 2018, 6, 154. [CrossRef] [PubMed]

17. Liu, H.; Archer, N.K.; Dillen, C.A.; Wang, Y.; Ashbaugh, A.G.; Ortines, R.V.; Kao, T.; Lee, S.K.; Cai, S.S.; Miller, R.J.; et al. Staphylococcus aureus Epicutaneous Exposure Drives Skin Inflammation via IL-36-Mediated T Cell Responses. Cell Host Microbe 2017, 22, 653-666.e5. [CrossRef]

18. Nakagawa, S.; Matsumoto, M.; Katayama, Y.; Oguma, R.; Wakabayashi, S.; Nygaard, T.; Saijo, S.; Inohara, N.; Otto, M.; Matsue, H.; et al. Staphylococcus aureus Virulent PSMalpha Peptides Induce Keratinocyte Alarmin Release to Orchestrate IL-17-Dependent Skin Inflammation. Cell Host Microbe 2017, 22, 667-677.e5. [CrossRef]

19. Noda, S.; Suarez-Farinas, M.; Ungar, B.; Kim, S.J.; de Guzman Strong, C.; Xu, H.; Peng, X.; Estrada, Y.D.; Nakajima, S.; Honda, T.; et al. The Asian atopic dermatitis phenotype combines features of atopic dermatitis and psoriasis with increased TH17 polarization. J. Allergy Clin. Immunol. 2015, 136, 1254-1264. [CrossRef]

20. Koga, C.; Kabashima, K.; Shiraishi, N.; Kobayashi, M.; Tokura, Y. Possible pathogenic role of Th17 cells for atopic dermatitis. J. Investig. Dermatol. 2008, 128, 2625-2630. [CrossRef] 
21. Joo, H.S.; Cheung, G.Y.; Otto, M. Antimicrobial activity of community-associated methicillin-resistant Staphylococcus aureus is caused by phenol-soluble modulin derivatives. J. Biol. Chem. 2011, 286, 8933-8940. [CrossRef] [PubMed]

22. Yang, J.J.; Chang, T.W.; Jiang, Y.; Kao, H.J.; Chiou, B.H.; Kao, M.S.; Huang, C.M. Commensal Staphylococcus aureus Provokes Immunity to Protect against Skin Infection of Methicillin-Resistant Staphylococcus aureus. Int. J. Mol. Sci. 2018, 19, 1290. [CrossRef] [PubMed]

23. Lai, Y.; Cogen, A.L.; Radek, K.A.; Park, H.J.; Macleod, D.T.; Leichtle, A.; Ryan, A.F.; Di Nardo, A.; Gallo, R.L. Activation of TLR2 by a small molecule produced by Staphylococcus epidermidis increases antimicrobial defense against bacterial skin infections. J. Investig. Dermatol. 2010, 130, 2211-2221. [CrossRef] [PubMed]

24. Lai, Y.; Di Nardo, A.; Nakatsuji, T.; Leichtle, A.; Yang, Y.; Cogen, A.L.; Wu, Z.R.; Hooper, L.V.; Schmidt, R.R.; von Aulock, S.; et al. Commensal bacteria regulate Toll-like receptor 3-dependent inflammation after skin injury. Nat. Med. 2009, 15, 1377-1382. [CrossRef] [PubMed]

25. Li, D.; Wang, W.; Wu, Y.; Ma, X.; Zhou, W.; Lai, Y. Lipopeptide 78 from Staphylococcus epidermidis Activates beta-Catenin To Inhibit Skin Inflammation. J. Immunol. 2019. [CrossRef] [PubMed]

26. Cogen, A.L.; Yamasaki, K.; Sanchez, K.M.; Dorschner, R.A.; Lai, Y.; MacLeod, D.T.; Torpey, J.W.; Otto, M.; Nizet, V.; Kim, J.E.; et al. Selective antimicrobial action is provided by phenol-soluble modulins derived from Staphylococcus epidermidis, a normal resident of the skin. J. Investig. Dermatol. 2010, 130, 192-200. [CrossRef] [PubMed]

27. Cogen, A.L.; Yamasaki, K.; Muto, J.; Sanchez, K.M.; Crotty Alexander, L.; Tanios, J.; Lai, Y.; Kim, J.E.; Nizet, V.; Gallo, R.L. Staphylococcus epidermidis antimicrobial delta-toxin (phenol-soluble modulin-gamma) cooperates with host antimicrobial peptides to kill group A Streptococcus. PLoS ONE 2010, 5, e8557. [CrossRef]

28. Nakatsuji, T.; Chen, T.H.; Butcher, A.M.; Trzoss, L.L.; Nam, S.J.; Shirakawa, K.T.; Zhou, W.; Oh, J.; Otto, M.; Fenical, W.; et al. A commensal strain of Staphylococcus epidermidis protects against skin neoplasia. Sci. Adv. 2018, 4, eaao4502. [CrossRef]

29. Tang, Y.; Chen, Y.; Jiang, H.; Robbins, G.T.; Nie, D. G-protein-coupled receptor for short-chain fatty acids suppresses colon cancer. Int. J. Cancer 2011, 128, 847-856. [CrossRef]

30. Wu, S.; Rhee, K.J.; Albesiano, E.; Rabizadeh, S.; Wu, X.; Yen, H.R.; Huso, D.L.; Brancati, F.L.; Wick, E.; McAllister, F.; et al. A human colonic commensal promotes colon tumorigenesis via activation of $\mathrm{T}$ helper type 17 T cell responses. Nat. Med. 2009, 15, 1016-1022. [CrossRef]

31. Bindels, L.B.; Porporato, P.; Dewulf, E.M.; Verrax, J.; Neyrinck, A.M.; Martin, J.C.; Scott, K.P.; Buc Calderon, P.; Feron, O.; Muccioli, G.G.; et al. Gut microbiota-derived propionate reduces cancer cell proliferation in the liver. Br. J. Cancer 2012, 107, 1337-1344. [CrossRef] [PubMed]

32. Naik, S.; Bouladoux, N.; Linehan, J.L.; Han, S.J.; Harrison, O.J.; Wilhelm, C.; Conlan, S.; Himmelfarb, S.; Byrd, A.L.; Deming, C.; et al. Commensal-dendritic-cell interaction specifies a unique protective skin immune signature. Nature 2015, 520, 104-108. [CrossRef] [PubMed]

33. Scharschmidt, T.C.; Vasquez, K.S.; Truong, H.A.; Gearty, S.V.; Pauli, M.L.; Nosbaum, A.; Gratz, I.K.; Otto, M.; Moon, J.J.; Liese, J.; et al. A Wave of Regulatory T Cells into Neonatal Skin Mediates Tolerance to Commensal Microbes. Immunity 2015, 43, 1011-1021. [CrossRef] [PubMed]

34. Volz, T.; Kaesler, S.; Draing, C.; Hartung, T.; Rocken, M.; Skabytska, Y.; Biedermann, T. Induction of IL-10-balanced immune profiles following exposure to LTA from Staphylococcus epidermidis. Exp. Dermatol. 2018, 27, 318-326. [CrossRef] [PubMed]

35. Otto, M. Staphylococcus epidermidis-the 'accidental' pathogen. Nat. Rev. Microbiol. 2009, 7, 555-567. [CrossRef] [PubMed]

36. Rogers, K.L.; Fey, P.D.; Rupp, M.E. Coagulase-negative staphylococcal infections. Infect. Dis. Clin. North. Am. 2009, 23, 73-98. [CrossRef] [PubMed]

37. Heilmann, C.; Ziebuhr, W.; Becker, K. Are coagulase-negative staphylococci virulent? Clin. Microbiol. Infect. 2018. [CrossRef]

38. Dong, Y.; Speer, C.P.; Glaser, K. Beyond sepsis: Staphylococcus epidermidis is an underestimated but significant contributor to neonatal morbidity. Virulence 2018, 9, 621-633. [CrossRef]

39. Nguyen, T.H.; Park, M.D.; Otto, M. Host Response to Staphylococcus epidermidis Colonization and Infections. Front. Cell. Infect. Microbiol. 2017, 7, 90. [CrossRef] 
40. Meric, G.; Mageiros, L.; Pensar, J.; Laabei, M.; Yahara, K.; Pascoe, B.; Kittiwan, N.; Tadee, P.; Post, V.; Lamble, S.; et al. Disease-associated genotypes of the commensal skin bacterium Staphylococcus epidermidis. Nat. Commun. 2018, 9, 5034. [CrossRef]

41. Hon, K.L.; Tsang, Y.C.; Pong, N.H.; Leung, T.F.; Ip, M. Exploring Staphylococcus epidermidis in atopic eczema: Friend or foe? Clin. Exp. Dermatol. 2016, 41, 659-663. [CrossRef] [PubMed]

42. Byrd, A.L.; Deming, C.; Cassidy, S.K.B.; Harrison, O.J.; Ng, W.I.; Conlan, S.; Program, N.C.S.; Belkaid, Y.; Segre, J.A.; Kong, H.H. Staphylococcus aureus and Staphylococcus epidermidis strain diversity underlying pediatric atopic dermatitis. Sci. Transl. Med. 2017, 9, eaal4651. [CrossRef] [PubMed]

43. Nakatsuji, T.; Chen, T.H.; Narala, S.; Chun, K.A.; Two, A.M.; Yun, T.; Shafiq, F.; Kotol, P.F.; Bouslimani, A.; Melnik, A.V.; et al. Antimicrobials from human skin commensal bacteria protect against Staphylococcus aureus and are deficient in atopic dermatitis. Sci. Transl. Med. 2017, 9, eaah4680. [CrossRef] [PubMed]

(C) 2019 by the authors. Licensee MDPI, Basel, Switzerland. This article is an open access article distributed under the terms and conditions of the Creative Commons Attribution (CC BY) license (http:/ / creativecommons.org/licenses/by/4.0/). 